\title{
Identification of Optical Counterparts of ULX sources
}

\author{
Carlos M. Gutiérrez and Martín López-Corredoira \\ Instituto de Astrofísica de Canarias, 38200 la Laguna, Tenerife, SPAIN \\ email: cgc@ll.iac.es, martinlc@ll.iac.es
}

\begin{abstract}
We present the results of an on-going program for the identification and characterization of optical counterparts of Ultra-Luminous X-ray (ULX) sources. The targets have been selected from the catalogues by Colbert \& Ptak (2002) and Swartz et al. (2004). A clear identification based on unambiguous optical spectral features was possible for 26 objects. A large number of objects result to be QSOs at higher redshift than the putative parent galaxy, and other ULXs seem to be associated to HII regions. In a few cases the optical counterpart results a foreground star in our galaxy. The observational program will continue to obtain a representative sample for statistical studies.
\end{abstract}

Keywords. X-rays: Ultra-luminous sources.

One of the most intriguing astrophysical objects are the ultra-luminous X-ray sources (ULXs) which have been discovered around nearby galaxies by the X-ray satellites Einstein, ROSAT, Chandra and XMM. Assuming that they are at the distance of their parent galaxies, their luminosities in the 0.1-2.4 Kev X-ray band are in the range $10^{39}-10^{40} \mathrm{erg} \mathrm{s}^{-1}$. Several explanations about the nature of these objects in terms of intermediate mass black holes associated with globular clusters, HII regions, supernova remnants, etc (Pakull \& Mirioni 2002; Angelini et al. 2001; Gao et al. 2003; Roberts et al. 2003; Wang 2002), local QSOs (Burbidge et al. 2003), hypothetical supermassive stars or beamed emission (King et al. 2001; Kording et al. 2002) have been proposed. To disentangle the nature of these objects it is important but still scarce the identification of optical counterparts (e. g. Roberts et al. 2001; Wu et al. 2002; Maseti et al. 2003; Liu et al. 2004). This motivated us to start this study searching for such optical counterparts in the Digital Sky Survey (DSS) images, in the digitalized USNO catalogues, and in the released Sloan Digital Sky Survey (SDSS) images. For instance, we have identified possible optical counterparts, in an error circle compatible with the X-ray position, for $\sim 50 \%$ of ULXs listed in Colbert \& Ptak (2002). The typical magnitudes of such objects are 18-20 in the $b$ band and are therefore bright enough targets for spectroscopic observations with 2 to $4 \mathrm{~m}$ telescopes.

Previously we have presented the identification of 9 of such optical counterparts (Arp et al. 2004; Gutiérrez \& López-Corredoira 2005) showing that 8 of these sources correspond to QSOs at higher redshift than their putative parent galaxy. Another object was associated with an HII region at the redshift of the parent galaxy (NGC 1073). Here, we present 17 new identifications of such optical counterparts based on long-slit spectroscopic observations obtained in several runs at the William Herschel Telescope (WHT) in the Observatory Roque de los Muchachos, La Palma, and at the $1.93 \mathrm{~m}$ telescope in the Haute Provence Observatory (OHP). The typical slit width was 2 arcsec, and the resolution $\sim 3-6 \AA$. Exposure times were between 900 and $1800 \mathrm{sec}$ for WHT observations, and between 1800 and $5400 \mathrm{sec}$ for observations at OHP. The spectra were 


\begin{tabular}{llllrrrrc}
\hline ID & Obs. & Galaxy & $z_{\text {gal }}$ & $\begin{array}{r}D_{g a l} \\
(\mathrm{Mpc})\end{array}$ & $\begin{array}{r}\log \left(L_{X}\right) \\
\left(\mathrm{erg} \mathrm{s}^{-1}\right)\end{array}$ & $\begin{array}{r}\text { Sep } \\
\left({ }^{\prime}\right)\end{array}$ & $z_{U L X}$ & Type \\
\hline IXO 22 & OHP Mar 2005 IC 342 & 0.0001 & 3.90 & 39.2 & 5.1 & 0.00 & HII region \\
IXO 31 & WHT Feb 2004 & Holmberg II & 0.0005 & 4.50 & 40.2 & 2.1 & 0.0009 & HII region (or M star) \\
IXO 32 & WHT Feb 2004 & NGC 2775 & 0.0045 & 18.05 & 39.2 & 3.7 & 2.770 & QSO \\
IXO 34 & WHT Feb 2004 & NGC 3031 & -0.0001 & 3.55 & 40.0 & 12.5 & 0.001 & HII region \\
IXO 35 & WHT Feb 2004 & NGC 3226 & 0.0038 & 17.63 & 39.2 & 2.4 & & M star \\
IXO 37 & WHT Feb 2004 & IC 2597 & 0.0076 & 40.09 & 40.9 & 1.9 & 0.567 & ELG \\
IXO 40 & WHT Feb 2004 & NGC 3923 & 0.0058 & 22.24 & 39.9 & 4.4 & 0.787 & QSO \\
IXO 43 & OHP Mar 2005 & NGC 4151 & 0.0033 & 20.30 & 39.3 & 5.0 & 0.24 & ELG \\
IXO 54 & OHP Mar 2005 & NGC 4438 & 0.0002 & 16.80 & 39.9 & 3.5 & 0.66 & QSO \\
IXO 57 & OHP Mar 2005 & NGC 4472 & 0.0033 & 16.80 & 39.8 & 8.1 & 0.85 & QSO \\
IXO 65 & OHP Mar 2005 & NGC 4559 & 0.0027 & 9.70 & 39.9 & 2.0 & 0.0027 & HII region \\
IXO 70 & OHP Mar 2005 & NGC 4649 & 0.0037 & 14.85 & 39.3 & 7.0 & 0.62 & QSO \\
SW 38 & OHP Mar 2005 & NGC 3079 & 0.0037 & 15.51 & 39.5 & 1.9 & 0.004 & HII region \\
SW 44 & OHP Mar 2005 & NGC 3556 & 0.0023 & 9.67 & 39.6 & 0.7 & & star \\
SW 45 & OHP Mar 2005 & NGC 3556 & 0.0023 & 9.67 & 39.2 & 0.1 & 0.0023 & HII region \\
SW 90 & OHP Mar 2005 & NGC 4490 & 0.0019 & 7.80 & 39.4 & 1.2 & 0.0015 & HII region \\
SW 134 & OHP Mar 2005 & NGC 5457 & 0.0008 & 7.24 & 38.9 & 6.2 & & M star
\end{tabular}

analyzed following a standard procedure using IRAF $\dagger$. The Table presents a summary of our results (another four objects were observed but the optical spetra did not allow a clear characterization). The columns are: (1): name of the ULX (IXO or SW for objects from the Colbert \& Ptak (2002) or Swartz et al. (2004) catalogues respectively). (2): telescope and epoch of observation. (3)-(5): name, redshift and distance of the parent galaxy. (6): X-ray luminosity assuming that the ULXs are at the distance of the parent galaxy. (7): projected distance between the ULXs and their parent galaxy. (8)-(9): redshift and nature of the optical counterpart of the ULXs.

\section{References}

Angelini, L., Loewenstein, M. \& Mushotzky, R. F. 2001, ApJ 557, L35

Arp, H., Gutiérrez, C. M. \& López-Corredoira, M. 2004, A\&̊A 418, 877

Burbidge, G., Burbidge, E. M. \& Arp, H. 2003, A\&A 400, L17

Colbert, E. \& Ptak, A. 2002, ApJS 143, 25

Gao, Y., Wang, Q. D., Appleton, P. N. \& Lucas, R. A. 2003, ApJ 596, L171

Gutiérrez, C. M. \& López-Corredoira, M. 2005, ApJ 622, L89

King, A. R., Davies, M. B., Ward, M. J., Fabbiano, G. \& Elvis, M. 2001, ApJ 552, L109

Kording, E., Falcke, H. \& Markoff, S. 2002, A\&A 382, L13

Liu, J. F., Bregman, J. N. \& Seitzer, P. 2004, ApJ 602, 249

Maseti, N. et al. 2003, A\&GA 406, L27

Pakull, M. W. \& Mirioni, L. 2002, in New Visions of the X-ray Universe in the XMM-Newton and Chandra Era, ESTEC

Roberts, T. P. et al. 2001, MNRAS 325, L7

Roberts, T. P., Goad, M. R. \& Warwick, R. S. 2003, MNRAS 342, 709

Swartz, D. A., Ghosh, K. K., Tennant, A. F. \& Wu, K. 2004, ApJS 154, 519

Wang, Q. D. 2002, MNRAS 332, 764

Wu, H., Xue, S. J., Xia, X. Y., Deng, Z. G. \& Mao, S. 2002, ApJ 576, 738

$\dagger$ IRAF is the Image Reduction and Analysis Facility, written and supported by the IRAF programming group at the National Optical Astronomy Observatory (NOAO) in Tucson, Arizona. 


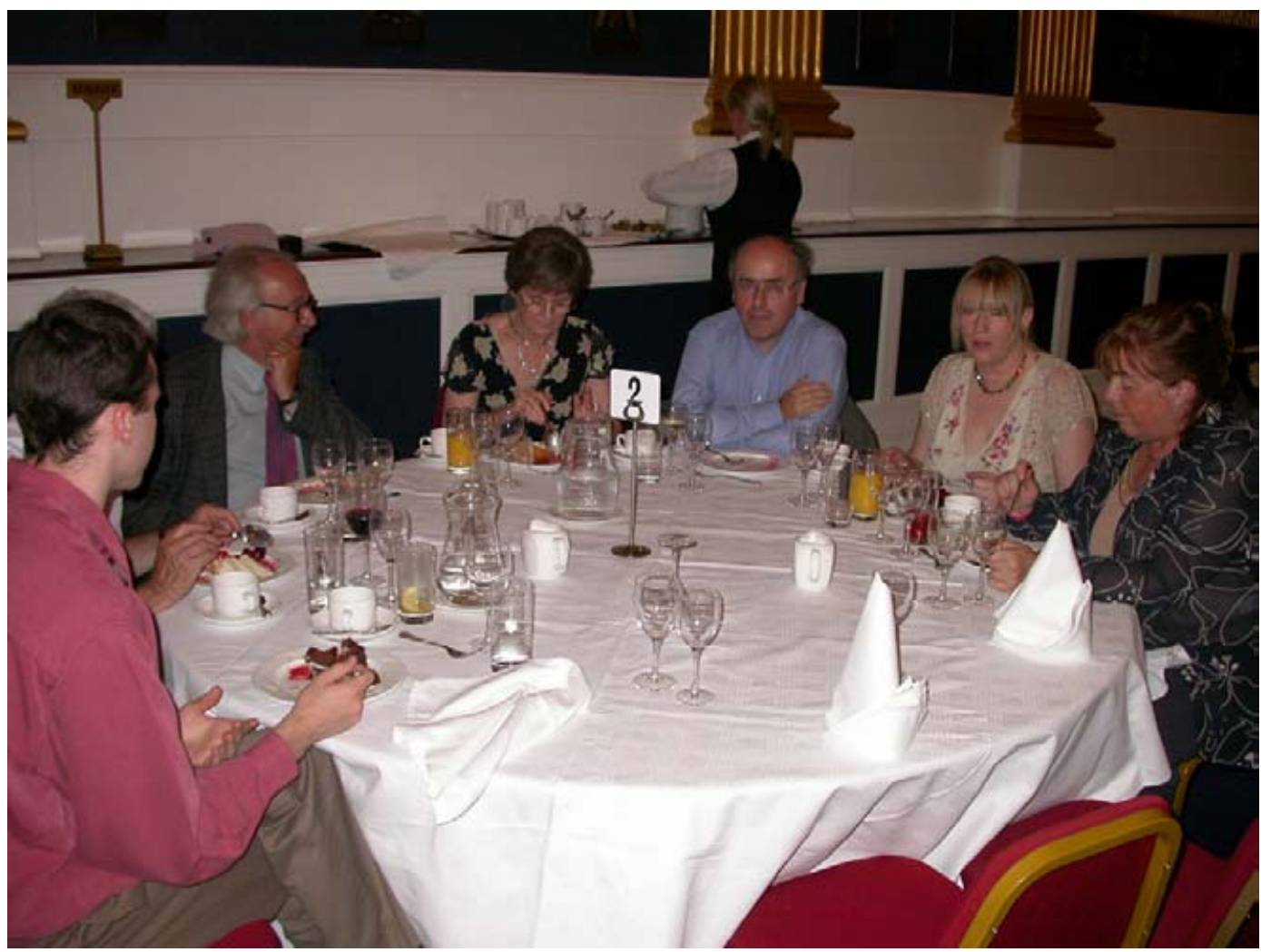

Francesco Polcaro tries to follow several Irish dialects. From left to right: Cóilín Ó Maoiléidigh, Brendan Jordan (hands + dessert only), Francesco Polcaro, Catherine Handley, Eamonn Cunningham, Carol Woods (who had avoided being photographed when the Conference Photo was taken), Hilary O'Donnell. 